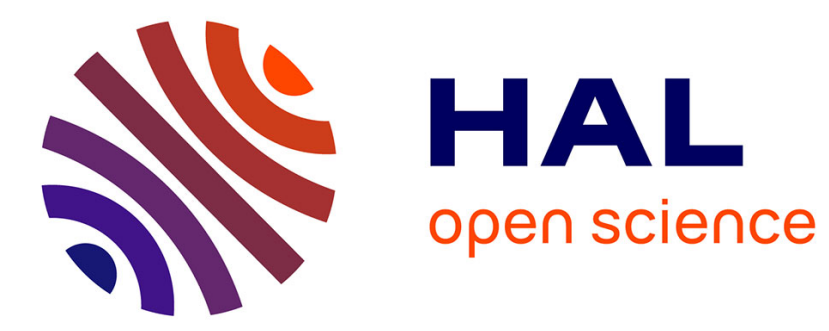

\title{
Central limit theorems for a branching random walk with a random environment in time
}

\author{
Zhiqiang Gao, Quansheng Liu, Hesong Wang
}

\section{To cite this version:}

Zhiqiang Gao, Quansheng Liu, Hesong Wang. Central limit theorems for a branching random walk with a random environment in time. Acta Mathematica Scientia, 2014, 34 (2), pp.501-512. 10.1016/S0252-9602(14)60023-0 . hal-00907152

\section{HAL Id: hal-00907152 \\ https://hal.science/hal-00907152}

Submitted on 21 Nov 2013

HAL is a multi-disciplinary open access archive for the deposit and dissemination of scientific research documents, whether they are published or not. The documents may come from teaching and research institutions in France or abroad, or from public or private research centers.
L'archive ouverte pluridisciplinaire HAL, est destinée au dépôt et à la diffusion de documents scientifiques de niveau recherche, publiés ou non, émanant des établissements d'enseignement et de recherche français ou étrangers, des laboratoires publics ou privés. 


\title{
Central limit theorems for a branching random walk in a random environment
}

\author{
Zhiqiang GAO* Quansheng LIU $^{\dagger} \quad$ Hesong WANG ${ }^{\ddagger}$
}

May 6, 2012

\begin{abstract}
We consider a branching random walk with a random environment in time, in which the offspring distribution of a particle of generation $n$ and the distribution of the displacements of its children depend on an environment indexed by the time $n$. The environment is supposed to be stationary and ergodic. For $A \subset \mathbb{R}$, let $Z_{n}(A)$ be the number of particles of generation $n$ located in $A$. We show central limit theorems for the counting measure $Z_{n}(\cdot)$ with appropriate normalization.
\end{abstract}

2000 Mathematics Subject Classification. 60J10, 60F05, 60J80.

Key Words and phrases. Branching random walk, random environment in time, central limit theorems.

\section{Introduction}

The model of branching random walks has been well studied in the literature: see e.g. [1, 5, 6, 12, 17 , and the reference therein. In the classical branching random walk, the point processes indexed by the particles $u$, formulated by the number of its children and their displacements, have a fixed constant distribution for all particles $u$. In reality these distributions may vary from generation to generation according to a random environment, just as in the case of a branching process in a random environment introduced in [2, 3, 18. In other words, the distributions themselves may be realizations of a stochastic process, rather than being fixed. Here, we study such a model which we call a branching random walk with a random environment in time. In this model, the offspring distributions of a particle of generation $n$ and the distributions of the displacements of its children depend on an environment indexed by $n$. This model was first introduced by Biggins and Kyprianou (2004, 7]). It is different to the usual branching random walks in a random environment (see e.g. [4, 9]), in which the authors considered the case where the offspring distribution of a particle situated at $z \in \mathbb{R}$ depends on a random environment indexed by $z$, while the moving mechanism is controlled by a fixed deterministic law.

\footnotetext{
*School of Mathematical Sciences, Beijing Normal University, Laboratory of Mathematics and Complex Systems, 100875 Beijing, China (gaozq@bnu.edu.cn) and L.M.A.M. Université de Bretagne Sud, campus de Tohannic, BP 573, 56017 Vannes, France.

${ }^{\dagger}$ Corresponding author, L.M.A.M. Université de Bretagne Sud, campus de Tohannic, BP 573, 56017 Vannes, France (quansheng.liu@univ-ubs.fr).

$\ddagger$ College of Mathematics and Computer Science, Hunan Normal University and College of Mathematics and Computing Science, Changsha University of Science and Technology Changsha, 410076 Hunan, People's Republic of China (hesongwang@tom.com).
} 
Some limit theorems on this model were announced in Liu (2007, 15]). Here we are interested in central limit theorems for the counting measure $Z_{n}(\cdot)$ which counts the number of particles of generation $n$ situated in a given set. For the classical branching random walk, such central limit theorems were proved by Kaplan and Assmussen (1976, [12]), which confirms a conjecture of Harris in 1963 ([10]). For more results on this subject, see e.g. Kaplan (1982, [1] ), Klebaner (1982, [13]) and Biggins (1990, 6] ).

The main objective of the present paper is to extend the basic results of Kaplan and Assmussen (1976, [12]) to the random environment case. The main results will be stated in Section 2, while their proofs will be given in Sections $3 \sim 5$.

\section{Description of the model and the main results}

\subsection{Description of the model}

A random environment in time $\xi=\left(\xi_{n}\right)$ is formulated as a sequence of random variables independent and identically distributed with values in some measurable space $(\Theta, \mathcal{F})$. Each realization of $\xi_{n}$ corresponds to two probability distributions $p\left(\xi_{n}\right)$ and $G_{\xi_{n}}$, where $p\left(\xi_{n}\right)=\left\{p_{k}\left(\xi_{n}\right): k \in \mathbb{N}\right\}$ is a probability law on $\mathbb{N}$ and $G_{\xi_{n}}$ a probability law on $\mathbb{R}$. Without loss of generality, we can take $\xi_{n}$ as coordinate functions defined on the product space $\left(\Theta^{\mathbb{N}}, \mathcal{F}^{\otimes \mathbb{N}}\right)$, equipped with a probability law $\tau$ which is invariant and ergodic under the usual shift transformation $\theta$ on $\Theta^{\mathbb{N}}$ : $\theta\left(\xi_{0}, \xi_{1}, \cdots\right)=\left(\xi_{1}, \xi_{2}, \cdots\right)$.

When the environment $\xi=\left(\xi_{n}\right)$ is given, the process can be described as follows. The process begins at time 0 with one initial particle $\varnothing$ of generation 0 located at $S_{\varnothing}=0 \in \mathbb{R}$; at time 1 , it is replaced by $N=N_{\varnothing}$ new particles of generation 1 , located at $L_{i}=L_{\varnothing i}, 1 \leq i \leq N$, where $N$ is of distribution $p\left(\xi_{0}\right)$ and each $L_{i}$ is of distribution $G_{\xi_{0}}$, given the environment $\xi$. In general, each particle $u=u_{1} \ldots u_{n}$ of generation $n$ is replaced at time $n+1$ by $N_{u}$ new particles of generation $n+1$, with displacements $L_{u 1}, L_{u 2}, \cdots, L_{u N_{u}}$, so that the $i$-th child is located at

$$
S_{u i}=S_{u}+L_{u i}
$$

where $N_{u}$ is of distribution $p\left(\xi_{n}\right)$ and each $L_{u i}$ is of distribution $G_{\xi_{n}}$, given the environment $\xi$. All the random variables $N_{u}$ and $L_{u}$, indexed by all finite sequences $u$ of positive integers, are independent of each other, given the environment $\xi$. We abbreviate $G_{\xi_{n}}$ as $G_{n}$ in the rest of this chapter.

Let $\left(\Gamma, \mathbb{P}_{\xi}\right)$ be the probability space under which the process is defined when the environment $\xi$ is fixed. As usual, $\mathbb{P}_{\xi}$ is called quenched law. The total probability space can be formulated as the product space $\left(\Gamma \times \Theta^{\mathbb{N}}, \mathbb{P}\right)$, where $\mathbb{P}=\mathbb{P}_{\xi} \otimes \tau$ in the sense that for all measurable and positive function $g$, we have

$$
\int g d \mathbb{P}=\iint g(y, \xi) d \mathbb{P}_{\xi}(y) d \tau(\xi),
$$

(recall that $\tau$ is the law of the environment $\xi$ ). The probability $\mathbb{P}$ is called annealed law. The quenched law $\mathbb{P}_{\xi}$ may be viewed as the conditional probability of the annealed law $\mathbb{P}$ given $\xi$. We will use $\mathbb{E}_{\xi}$ to denote the expectation with respect to $\mathbb{P}_{\xi}$. Other expectations will be denoted simply $\mathbb{E}$ (there will be no confusion according to the context).

Let $\mathbb{T}$ be the genealogical tree with $\left\{N_{u}\right\}$ as defining elements. By definition, we have: (a) $\varnothing \in \mathbb{T}$; (b) $u i \in \mathbb{T}$ implies $u \in \mathbb{T}$; (c) if $u \in \mathbb{T}$, then $u i \in \mathbb{T}$ if and only if $1 \leq i \leq N_{u}$. Let $\mathbb{T}_{n}=\{u \in \mathbb{T}:|u|=n\}$ be the set of particles of generation $n$, where $|u|$ denotes the length of the sequence $u$ and represents the number of generation to which $u$ belongs. 


\subsection{The main results}

Let $Z_{n}(\cdot)$ be the counting measure of particles of generation $n$ : for $B \subset \mathbb{R}$,

$$
Z_{n}(B)=\sum_{u \in \mathbb{T}_{n}} \mathbf{1}_{B}\left(S_{u}\right)
$$

Then $\left\{Z_{n}(\mathbb{R})\right\}$ constitute a branching process in a random environment (see e.g. [2, 3, 18]).

For $n \geq 0$, define

$$
\begin{aligned}
m_{n} & =m_{n}(\xi)=\sum_{k=1}^{\infty} k p_{k}\left(\xi_{n}\right) \\
l_{n} & =l_{n}(\xi)=\int x \mathrm{~d} G_{n}(x), \quad \sigma_{n}^{2}=\sigma_{n}^{2}(\xi)=\int x^{2} \mathrm{~d} G_{n}(x)-l_{n}^{2} .
\end{aligned}
$$

Throughout the paper, we consider the supercritical case where

$$
\mathbb{E} \ln m_{0}>0 \quad \text { and } \quad \mathbb{E}\left(\frac{1}{m_{0}} \sum_{k=2}^{\infty}(k \ln k) p_{k}\left(\xi_{0}\right)\right)<\infty
$$

For simplicity, we assume that

$$
p_{0}\left(\xi_{0}\right)=0 \quad \text { a.s. }
$$

We also need the following assumptions:

$$
\mathbb{E} l_{0}<+\infty \text { and } 0<\mathbb{E} \sigma_{0}^{2}<+\infty \text {. }
$$

The following the $\sigma$-fields will be used: for $n \geq 1$,

$$
\begin{aligned}
\mathscr{F}_{n} & =\sigma\left(\xi, N_{u}:|u|<n\right), \\
\mathscr{D}_{n} & =\sigma\left(\xi, N_{u}, L_{u i}: i \geq 1,|u|<n\right), \\
\mathscr{I}_{n} & =\sigma\left(\xi_{k}, N_{u}, L_{u i}: k<n, i \geq 1,|u|<n\right) .
\end{aligned}
$$

We introduce the notations:

$$
\begin{aligned}
& \ell_{n}=\ell_{n}(\xi)=l_{0}+l_{1}+\cdots+l_{n-1}, \quad s_{n}^{2}=s_{n}^{2}(\xi)=\sigma_{0}^{2}+\sigma_{0}^{2}+\cdots+\sigma_{n-1}^{2}, \\
& m_{n}(2)=m_{n}(2, \xi)=\sum_{k=1}^{\infty} k^{2} p_{k}\left(\xi_{n}\right), \quad \gamma_{n}=\gamma_{n}(\xi)=\left(m_{n}(2)-m_{n}^{2}\right)^{1 / 2}, \\
& \pi_{0}=1 \quad \text { and } \quad \pi_{n}=\pi_{n}(\xi)=m_{0} \cdots m_{n-1} \quad \text { for } \quad n \geq 1 .
\end{aligned}
$$

It is well known that

$$
W_{n}=\left(\pi_{n}\right)^{-1} Z_{n}(\mathbb{R}), \quad n \geq 1
$$

constitute a martingale with respect to $\mathscr{F}_{n}$. Under (2.1), the limit

$$
W=\lim _{n} W_{n}
$$

exists a.s. with $\mathbb{E} W=1$ (see e.g. [3]); by 2.2 ,,$W>0$ a.s..

Now our main results can be formulated as follows.

Theorem 2.1. Assume that 2.1 - 2.3 hold. Then for all $t \in \mathbb{R}$, as $n \rightarrow \infty$,

$$
\left(\pi_{n}\right)^{-1} Z_{n}\left(\ell_{n}+s_{n} t\right) \rightarrow \Phi(t) W \quad \text { in probability }
$$

where $Z_{n}(x)=Z_{n}((-\infty, x])$ for $x \in \mathbb{R}$ and $\Phi(t)=\int_{-\infty}^{t}(2 \pi)^{-1 / 2} e^{-x^{2} / 2} \mathrm{~d} x$. 
Under some additional moment conditions, the convergence in probability can be improved to the a.s. convergence.

Theorem 2.2. Assume that 2.1 -2.3 hold and that for some $\eta>0$,

$$
\mathbb{E}\left[\frac{1}{m_{0}} \sum_{k=2}^{\infty} k(\ln k)^{2+\eta} p_{k}\left(\xi_{0}\right)\right]<+\infty,
$$

then it is almost sure that for all $t \in \mathbb{R}$, as $n \rightarrow \infty$,

$$
\left(\pi_{n}\right)^{-1} Z_{n}\left(\ell_{n}+s_{n} t\right) \rightarrow \Phi(t) W
$$

Also by a theorem of Klebaner (1982, [13), we can prove the following a.s. convergence under another additional moment condition. We should note the condition (2.7) here and the above one 2.5) do not imply each other.

Theorem 2.3. Assume that 2.1] -2.3 hold and that

$$
\mathbb{E} \ln ^{+} \frac{\gamma_{0}^{2}}{m_{0}^{2}}<+\infty
$$

then it is almost sure that for all $t \in \mathbb{R}$, as $n \rightarrow \infty$,

$$
\left(\pi_{n}\right)^{-1} Z_{n}\left(\ell_{n}+s_{n} t\right) \rightarrow \Phi(t) W
$$

Remark 2.4. By the monotonicity of $Z_{n}(x)$ and the continuity of $\Phi(x)$, it can be easily seen that 2.4 implies that for all $t \in \mathbb{R}$, as $n \rightarrow \infty$,

$$
\left(\pi_{n}\right)^{-1} Z_{n}\left(\ell_{n}+s_{n} t+o(\sqrt{n})\right) \rightarrow \Phi(t) W \quad \text { in probability. }
$$

Here we have use the fact that $s_{n} \sim \sqrt{n} \sigma$ (where $\sigma=\sqrt{\mathbb{E} \sigma_{0}^{2}}$ ). By the same reason, 2.4 (or 2.9) implies that for all $t \in \mathbb{R}$, as $n \rightarrow \infty$,

$$
\left(\pi_{n}\right)^{-1} Z_{n}\left(\ell_{n}+\sqrt{n} \sigma t+o(\sqrt{n})\right) \rightarrow \Phi(t) W \quad \text { in probability. }
$$

Similarly, 2.6 (or 2.8) implies that a.s. for all $t \in \mathbb{R}$, as $n \rightarrow \infty$,

$$
\left(\pi_{n}\right)^{-1} Z_{n}\left(\ell_{n}+\sqrt{n} \sigma t+o(\sqrt{n})\right) \rightarrow \Phi(t) W .
$$

For the classical branching random walk, 2.10 reduces to the result of Kaplan and Asmussen 12 .

We believe that it is possible to relax the moment condition (2.7) for the a.s. result (2.8) and extend the result to the more general case where the displacements of the children of the same parent can have different distributions. This is true for the deterministic case: see e.g. Kaplan (1982, [1]) and Biggins (1990, [6]).

\section{Proof of Theorem 2.1}

\subsection{Notations and a key decomposition}

We first introduce some notations. As usual, we write $\mathbb{N}^{*}=\{1,2,3, \cdots\}$ and denote by

$$
U=\bigcup_{n=0}^{\infty}\left(\mathbb{N}^{*}\right)^{n}
$$

the set of all finite sequences, where $\left(\mathbb{N}^{*}\right)^{0}=\{\varnothing\}$ contains the null sequence $\varnothing$. 
For all $u \in U$, let $\mathbb{T}(u)$ be the shifted tree of $\mathbb{T}$ at $u$ with defining elements $\left\{N_{u v}\right\}$ : we have 1 ) $\varnothing \in \mathbb{T}(u), 2) v i \in \mathbb{T}(u) \Rightarrow v \in \mathbb{T}(u)$ and 3$)$ if $v \in \mathbb{T}(u)$, then $v i \in \mathbb{T}(u)$ if and only if $1 \leq i \leq N_{u v}$. Define $\mathbb{T}_{n}(u)=\{v \in \mathbb{T}(u):|v|=n\}$. Recall that $\mathbb{T}=\mathbb{T}(\varnothing)$ and $\mathbb{T}_{n}=\mathbb{T}_{n}(\varnothing)$.

For $u \in\left(\mathbb{N}^{*}\right)^{k}(k \geq 0)$ and $n \geq 1$, let $S_{u}$ be the position of $u$ and write

$$
Z_{n}(u, B)=\sum_{v_{1} \cdots v_{n} \in \mathbb{T}_{n}(u)} \mathbf{1}_{B}\left(L_{u v_{1}}+\cdots+L_{u v_{1} \cdots v_{n}}\right),
$$

then the law of $Z_{n}(u, B)$ under $\mathbb{P}_{\xi}$ is that of $Z_{n}(B)$ under $P_{\theta^{k}}$. Define

$$
\begin{aligned}
& W_{n}(u, B)=\left(\pi_{n}\left(\theta^{k} \xi\right)\right)^{-1} Z_{n}(u, B), \quad W_{n}(u, t)=W_{n}(u,(-\infty, t]), \\
& W_{n}(B)=\left(\pi_{n}\right)^{-1} Z_{n}(B), \quad W_{n}(t)=W_{n}((-\infty, t]) .
\end{aligned}
$$

By definition, $\pi_{n}\left(\theta^{k} \xi\right)=m_{k} \cdots m_{k+n-1}, Z_{n}(B)=Z_{n}(\varnothing, B)$ ( $\varnothing$ is the initial particle), $W_{n}(B)=$ $W_{n}(\varnothing, B), W_{n}=W_{n}(\mathbb{R})$ and fixed the environment $\xi$, for $k \leq n$,

$$
Z_{n}(B)=\sum_{u \in \mathbb{T}_{k}} Z_{n-k}\left(u, B-S_{u}\right) .
$$

Notice that for $u \in U$,

$$
Z_{n-k}\left(u, B-S_{u}\right)=\sum_{v_{1} \cdots v_{n-k} \in \mathbb{T}_{n-k}(u)} \mathbf{1}_{B}\left(S_{u v_{1} \cdots v_{n-k}}\right)
$$

represents the descendants of $u$ at time $n$ situated in $B$.

Hereafter, we will use the following notations for the convention:

$$
\mathbb{P}_{\xi, n}(\cdot)=\mathbb{P}_{\xi}\left(\cdot \mid \mathscr{I}_{n}\right), \quad \mathbb{P}_{n}(\cdot)=\mathbb{P}\left(\cdot \mid \mathscr{I}_{n}\right) ; \quad \mathbb{E}_{\xi, n}(\cdot)=\mathbb{E}_{\xi}\left(\cdot \mid \mathscr{I}_{n}\right), \quad \mathbb{E}_{n}(\cdot)=\mathbb{E}\left(\cdot \mid \mathscr{I}_{n}\right) .
$$

For each $n$, we choose an integer $k_{n}<n$ as follows. Let $\beta$ with $1 / 2<\beta<1$ and $\alpha>2 /\left(\beta^{-1}-1\right)$. For $j \in \mathbb{N}$ and $j^{\alpha / \beta} \leq n<(j+1)^{\alpha / \beta}$, set $k_{n}=a_{j}=\left[j^{\alpha}\right]$. Let $t_{n}=\ell_{n}+s_{n} t$ for $t \in \mathbb{R}$ and $n \geq 1$. Then by 3.1,

$$
W_{n}\left(t_{n}\right)=A_{n}+B_{n}+W_{k_{n}} \Phi(t)
$$

where

$$
\begin{aligned}
& A_{n}=\frac{1}{\pi_{k_{n}}} \sum_{u \in \mathbb{T}_{k_{n}}}\left\{W_{n-k_{n}}\left(u, t_{n}-S_{u}\right)-\mathbb{E}_{\xi, k_{n}}\left(W_{n-k_{n}}\left(u, t_{n}-S_{u}\right)\right)\right\}, \\
& B_{n}=\frac{1}{\pi_{k_{n}}} \sum_{u \in \mathbb{T}_{k_{n}}}\left[\mathbb{E}_{\xi, k_{n}}\left(W_{n-k_{n}}\left(u, t_{n}-S_{u}\right)\right)-\Phi(t)\right] .
\end{aligned}
$$

Here we remind that the random variables $W_{n-k_{n}}\left(u, t_{n}-S_{u}\right)$ are independent of each other under the conditional probability $\mathbb{P}_{\xi, k_{n}}$.

For the proof of Theorem 2.1] as in Asmussen and Kaplan [12, the main idea is to use the decomposition formula 3.2 , proving that $A_{n} \rightarrow 0$ and $B_{n} \rightarrow 0$ in probability. However, new ideas will be needed for the proof of the later due to the appearance of the random environment.

\subsection{Two lemmas}

Using the decomposition formula 3.2 , we proceed the proof of Theorem 2.1 by establishing two lemmas:

Lemma 3.1. If 2.1] holds, then

$$
A_{n} \rightarrow 0 \quad \text { in } \quad \text { probability under } \mathbb{P} \text {. }
$$


Lemma 3.2. If (2.3) holds, then

$$
B_{n} \rightarrow 0 \quad \mathbb{P} \text {-a.s. . }
$$

For the proof of Lemma 3.1, we shall use some method which is different from that of Kaplan and Asmussen [12].

Proof of Lemma 3.1. For convenience, we introduce the following notations: for $n \geq 1, u \in \mathbb{T}_{k_{n}}$,

$$
\begin{aligned}
& X_{n, u}=W_{n-k_{n}}\left(u, t_{n}-S_{u}\right)-\mathbb{E}_{\xi, k_{n}}\left(W_{n-k_{n}}\left(u, t_{n}-S_{u}\right), \quad Y_{n}=\frac{1}{Z_{k_{n}}} \sum_{u \in \mathbb{T}_{k_{n}}} X_{n, u} ;\right. \\
& \bar{X}_{n, u}=X_{n, u} \mathbf{1}_{\left\{\left|X_{n, u}\right|<Z_{k_{n}}\right\}}, \quad \bar{Y}_{n}=\frac{1}{Z_{k_{n}}} \sum_{u \in \mathbb{T}_{k_{n}}} \bar{X}_{n, u} .
\end{aligned}
$$

We shall prove that $\forall \varepsilon>0$,

$$
\mathbb{P}_{k_{n}}\left(\left|Y_{n}\right|>\varepsilon\right) \stackrel{n \rightarrow \infty}{\longrightarrow} 0 .
$$

From this and that $\frac{Z_{k_{n}}(\mathbb{R})}{\pi_{k_{n}}} \rightarrow W>0$ a.s., we shall have that $\forall \varepsilon>0$

$$
\mathbb{P}_{k_{n}}\left(\left|A_{n}\right|>\varepsilon\right) \stackrel{n \rightarrow \infty}{\longrightarrow} 0
$$

Now by the dominated convergence theorem, 3.3 follows.

We proceed the proof of (3.5) in 4 steps.

Observe that $\forall \varepsilon>0$,

$$
\begin{aligned}
\mathbb{P}_{k_{n}}\left(\left|Y_{n}\right|>2 \varepsilon\right) & \leq \mathbb{P}_{k_{n}}\left(\left|Y_{n}-\mathbb{E}_{\xi} \bar{Y}_{n}\right|>\varepsilon\right)+\mathbb{P}_{k_{n}}\left(\left|\mathbb{E}_{\xi} \bar{Y}_{n}\right|>\varepsilon\right) \\
& \leq \mathbb{P}_{k_{n}}\left(Y_{n} \neq \bar{Y}_{n}\right)+\mathbb{P}_{k_{n}}\left(\left|\bar{Y}_{n}-\mathbb{E}_{\xi} \bar{Y}_{n}\right|>\varepsilon\right)+\mathbb{P}_{k_{n}}\left(\left|\mathbb{E}_{\xi} \bar{Y}_{n}\right|>\varepsilon\right) .
\end{aligned}
$$

Step 1. We first prove that

$$
\mathbb{P}_{k_{n}}\left(Y_{n} \neq \bar{Y}_{n}\right) \stackrel{n \rightarrow \infty}{\longrightarrow} 0 .
$$

By the stationarity of the environment, we have

$$
\begin{aligned}
\mathbb{P}_{k_{n}}\left(Y_{n} \neq \bar{Y}_{n}\right) & \leq \sum_{u \in \mathbb{T}_{k_{n}}} \mathbb{P}_{k_{n}}\left(X_{n, u} \neq \bar{X}_{n, u}\right)=\sum_{u \in \mathbb{T}_{k_{n}}} \mathbb{P}_{k_{n}}\left(\left|X_{n, u}\right| \geq Z_{k_{n}}\right) \\
& \leq \sum_{u \in \mathbb{T}_{k_{n}}} \mathbb{P}_{k_{n}}\left(W_{n-k_{n}}(u)+1 \geq Z_{k_{n}}\right) \\
& =\left[r_{n} \mathbb{P}\left(W_{n-k_{n}}+1 \geq r_{n}\right)\right]_{r_{n}=Z_{k_{n}}} \\
& \leq\left[\mathbb{E}\left(\left(W_{n-k_{n}}+1\right) \mathbf{1}_{\left\{W_{n-k_{n}}+1 \geq r_{n}\right\}}\right)\right]_{r_{n}=Z_{k_{n}}} \stackrel{n \rightarrow \infty}{\longrightarrow} 0,
\end{aligned}
$$

where in the last step, we have used the facts that $\left\{W_{n-k_{n}}+1\right\}$ is uniformly integrable and $Z_{k_{n}} \rightarrow \infty$ a.s.. (The alert reader might have noticed that: $\left[r_{n} \mathbb{P}\left(W_{n-k_{n}}+1 \geq r_{n}\right)\right]_{r_{n}=Z_{k_{n}}} \neq Z_{k_{n}} \mathbb{P}\left(W_{n-k_{n}}+\right.$ $\left.1 \geq Z_{k_{n}}\right)$ ).

Step 2. Next we prove that

$$
\mathbb{P}_{k_{n}}\left(\left|\bar{Y}_{n}-\mathbb{E}_{\xi, k_{n}} \bar{Y}_{n}\right|>\varepsilon\right) \stackrel{n \rightarrow \infty}{\longrightarrow} 0 .
$$


Notice that

$$
\begin{aligned}
\mathbb{P}_{k_{n}}\left(\left|\bar{Y}_{n}-\mathbb{E}_{\xi, k_{n}} \bar{Y}_{n}\right|>\varepsilon\right) & =\mathbb{E}_{k_{n}}\left(\mathbb{P}_{\xi, k_{n}}\left(\left|\bar{Y}_{n}-\mathbb{E}_{\xi, k_{n}} \bar{Y}_{n}\right|>\varepsilon\right)\right) \\
& \leq \varepsilon^{-2} \mathbb{E}_{k_{n}}\left(Z_{k_{n}}^{-2} \sum_{u \in \mathbb{T}_{k_{n}}} \mathbb{E}_{\xi, k_{n}} \bar{X}_{n, u}^{2}\right) \\
& =\varepsilon^{-2} Z_{k_{n}}^{-2} \sum_{u \in \mathbb{T}_{k_{n}}} \mathbb{E}_{k_{n}} \bar{X}_{n, u}^{2}=\varepsilon^{-2} Z_{k_{n}}^{-2} \sum_{u \in \mathbb{T}_{k_{n}}} \mathbb{E}_{k_{n}}\left(X_{n, u}^{2} \mathbf{1}_{\left\{\left|X_{n, u}\right| \leq Z_{k_{n}}\right\}}\right) \\
& \leq \varepsilon^{-2} Z_{k_{n}}^{-2} \sum_{u \in \mathbb{T}_{k_{n}}} \mathbb{E}_{k_{n}}\left(\left(W_{n-k_{n}}(u)+1\right) \wedge Z_{k_{n}}\right)^{2} \\
& =\varepsilon^{-2}\left[\mathbb{E}_{n}^{-1}\left(\left(W_{n-k_{n}}+1\right) \wedge r_{n}\right)^{2}\right]_{r_{n}=Z_{k_{n}}}^{\stackrel{n \rightarrow \infty}{\longrightarrow} 0,}
\end{aligned}
$$

where in the last step, we have used the facts that $\left\{W_{n-k_{n}}+1\right\}$ is uniformly integrable, that the martingale $\left\{W_{n}\right\}$ converges to $W$ and that $Z_{k_{n}} \stackrel{n \rightarrow \infty}{\longrightarrow} \infty$ a.s..

Step 3. We then prove that

$$
\mathbb{P}_{k_{n}}\left(\left|\mathbb{E}_{\xi, k_{n}} \bar{Y}_{n}\right|>\varepsilon\right) \stackrel{n \rightarrow \infty}{\longrightarrow} 0 .
$$

By Chebyshev's inequality, we see that

$$
\begin{aligned}
\mathbb{P}_{k_{n}}\left(\left|\mathbb{E}_{\xi, k_{n}} \bar{Y}_{n}\right|>\varepsilon\right) & \leq \varepsilon^{-1} \mathbb{E}_{k_{n}}\left|\mathbb{E}_{\xi, k_{n}} \bar{Y}_{n}\right| \\
& \leq \varepsilon^{-1} Z_{k_{n}}^{-1} \sum_{u \in \mathbb{T}_{k_{n}}} \mathbb{E}_{k_{n}}\left|\mathbb{E}_{\xi, k_{n}} \bar{X}_{n, u}\right|=\varepsilon^{-1} Z_{k_{n}}^{-1} \sum_{u \in \mathbb{T}_{k_{n}}} \mathbb{E}_{k_{n}}\left|-\mathbb{E}_{\xi, k_{n}} X_{n, u} \mathbf{1}_{\left\{\left|X_{n, u}\right| \geq Z_{k_{n}}\right\}}\right| \\
& \leq \varepsilon^{-1} Z_{k_{n}}^{-1} \sum_{u \in \mathbb{T}_{k_{n}}} \mathbb{E}_{k_{n}}\left(\left|W_{n-k_{n}}(u)+1\right| \mathbf{1}_{\left\{\left|W_{n-k_{n}}(u)+1\right| \geq Z_{k_{n}}\right\}}\right) \\
& =\varepsilon^{-1}\left[\mathbb{E}\left(\left|W_{n-k_{n}}+1\right| \mathbf{1}_{\left\{\left|W_{n-k_{n}}+1\right| \geq r_{n}\right\}}\right)\right]_{r_{n}=Z_{k_{n}}} \rightarrow 0
\end{aligned}
$$

where in the last step, we have used the facts that $\left\{W_{n-k_{n}}+1\right\}$ is uniformly integrable and $Z_{k_{n}} \stackrel{n \rightarrow \infty}{\longrightarrow} \infty$ a.s..

Step 4. By (3.6), (3.7), (3.8) and (3.9), we see that $\forall \varepsilon>0, \mathbb{P}_{k_{n}}\left(\left|Y_{n}\right|>2 \varepsilon\right) \stackrel{n \rightarrow \infty}{\longrightarrow} 0$. Therefore (3.5) has been proved.

For the proof of Lemma 3.2 , we first prove the following result.

Lemma 3.3. Let $u \in \mathbb{T}_{k_{n}}$. For a.e. $\xi$,

$$
\begin{aligned}
& \mathbb{E}_{\xi} W_{n-k_{n}}(u, B)=G_{k_{n}} * \cdots * G_{n-1}(B), \quad u \in \mathbb{T}_{k_{n}} ; \\
& \mathbb{E}_{\xi} W_{n-k_{n}}\left(u, \widehat{t}_{n}\right) \rightarrow \Phi(t) \quad \text { uniformly in } t \text { as } n \rightarrow \infty
\end{aligned}
$$

where $\widehat{t}_{n}=\widehat{\ell}_{n-k_{n}}+\widehat{s}_{n-k_{n}} t, \widehat{\ell}_{n-k_{n}}=l_{k_{n}}+\cdots+l_{n-1}$ and $\widehat{s}_{n-k_{n}}^{2}=\sigma_{k_{n}}^{2}+\cdots+\sigma_{n-1}^{2}$.

Proof of Lemma 3.3. It is easy to check the first equality, which implies that the second assertion is equivalent to the following : for almost every $\xi$,

$$
G_{k_{n}} * \cdots * G_{n-1}\left(\widehat{t}_{n}\right) \rightarrow \Phi(t) \text { uniformly } \quad \text { in } \quad t \quad \text { as } n \rightarrow \infty \text {. }
$$

To prove this, we will use the Lindeberg-Feller theorem for an array of random variables, so it suffices to verify the following Lindeberg condition (see e.g. [16]) : for every $\varepsilon>0$,

$$
\lim _{n \rightarrow \infty} \frac{1}{\widehat{s}_{n-k_{n}}^{2}} \sum_{j=k_{n}}^{n} \int_{\left\{x:\left|x-l_{j}\right|>\varepsilon s_{n}\right\}}\left(x-l_{j}\right)^{2} d G_{j}(x)=0 \quad \text { a.s. }
$$


By the Birkhoff ergodic theorem,

$$
\lim _{n \rightarrow \infty} \frac{\widehat{s}_{n-k_{n}}^{2}}{n-k_{n}}=\lim _{n \rightarrow \infty} \frac{s_{n}^{2}-s_{k_{n}}^{2}}{n} \cdot \frac{n}{n-k_{n}}=\lim _{n \rightarrow \infty} \frac{s_{n}^{2}}{n}-\lim _{n \rightarrow \infty} \frac{k_{n}}{n} \frac{s_{k_{n}}^{2}}{k_{n}}=\mathbb{E} \sigma_{0}^{2} \quad \text { a.s. }
$$

and hence the above condition is equivalent to

$$
\lim _{n \rightarrow \infty} \frac{1}{n} \sum_{j=k_{n}}^{n} \int_{\left\{x:\left|x-l_{j}\right|>\varepsilon \sqrt{n}\right\}}\left(x-l_{j}\right)^{2} d G_{j}(x)=0 \quad \text { a.s. }
$$

To see that 3.12 holds, it suffices to notice that for all $K>0$ and $n>K$,

$$
\frac{1}{n} \sum_{j=k_{n}}^{n} \int_{\left\{x:\left|x-l_{j}\right|>\varepsilon \sqrt{n}\right\}}\left(x-l_{j}\right)^{2} d G_{j}(x) \leq \frac{1}{n} \sum_{j=1}^{n} \int_{\left\{x:\left|x-l_{j}\right|>\varepsilon \sqrt{K}\right\}}\left(x-l_{j}\right)^{2} d G_{j}(x) ;
$$

by the Birkhoff ergodic theorem, the right-hand side converges a.s. to

$$
\mathbb{E}\left\{\int_{\left\{x:\left|x-l_{0}\right|>\varepsilon \sqrt{K}\right\}}\left(x-l_{0}\right)^{2} d G_{0}(x)\right\},
$$

which can be as small as we want when $K$ is large enough . Thus 3.12 holds and 3.10 follows. So the lemma has been proved.

Proof of Lemma 3.2. We begin with the fact that for almost every environment $\xi$ and $u \in \mathbb{T}_{k_{n}}$,

$$
\mathbb{E}_{\xi, k_{n}}\left(W_{n-k_{n}}\left(u, t_{n}-S_{u}\right)\right)=G_{k_{n}} * \cdots * G_{n-1}\left(t_{n}-S_{u}\right) .
$$

By Lemma 3.3, we see that

$$
\sup _{x \in \mathbb{R}}\left|\Phi(x)-G_{k_{n}} * \cdots * G_{n-1}\left(x \widehat{s}_{n-k_{n}}+\widehat{\ell}_{n-k_{n}}\right)\right| \rightarrow 0 \quad \text { as } n \rightarrow \infty .
$$

Combining this with the fact that $t_{n}-S_{u}=\widehat{s}_{n-k_{n}} t+\widehat{\ell}_{n-k_{n}}+o(\sqrt{n})$, we see that a.s.,

$$
D_{n-k_{n}}(\xi)=\sup _{t \in \mathbb{R}}\left|\Phi(t)-G_{k_{n}} * \cdots * G_{n-1}\left(t_{n}-S_{u}\right)\right| \rightarrow 0 \quad \text { as } n \rightarrow \infty .
$$

Notice that

$$
\begin{aligned}
\left|B_{n}\right| & =\left|\left(\pi_{k_{n}}\right)^{-1} \sum_{u \in \mathbb{T}_{k_{n}}}\left\{\mathbb{E}_{\xi, k_{n}}\left(W_{n-k_{n}}\left(u, t_{n}-S_{u}\right)\right)-\Phi(t)\right\}\right| \\
& =\left(\pi_{k_{n}}\right)^{-1}\left|\sum_{u \in \mathbb{T}_{k_{n}}} G_{k_{n}} * G_{k_{n}+1} * \cdots * G_{n-1}\left(t_{n}-S_{u}\right)-\Phi(t)\right| \\
& \leq W_{k_{n}} D_{n-k_{n}}(\xi)+\left(\pi_{k_{n}}\right)^{-1} \sum_{u \in \mathbb{T}_{k_{n}}}\left|\Phi\left(\frac{t_{n}-S_{u}-\left(\ell_{n}-\ell_{k_{n}}\right)}{\sqrt{s_{n}^{2}-s_{k_{n}}^{2}}}\right)-\Phi(t)\right| \\
& \leq W_{k_{n}} D_{n-k_{n}}(\xi)+W_{k_{n}}\left|\Phi\left(\frac{s_{n} t}{\sqrt{s_{n}^{2}-s_{k_{n}}^{2}}}\right)-\Phi(t)\right|+\frac{C}{\pi_{k_{n}}} \sum_{u \in \mathbb{T}_{k_{n}}} \frac{\left|S_{u}-\ell_{k_{n}}\right|}{\sqrt{s_{n}^{2}-s_{k_{n}}^{2}}}
\end{aligned}
$$

where $C$ is a suitable constant independent of $\xi$. The last inequality follows from elementary calculus. In the last expression, the first term tends to 0 a.s. by (3.14 and the a.s. convergence of $W_{n}$; it is clear that the second term tends to 0 a.s.. So the lemma will be proved once we prove that the third term tends to 0 a.s.. In view of the definition of $k_{n}$, it suffices to show that

$$
M_{j}=\left(\pi_{a_{j}}\right)^{-1} \sum_{u \in \mathbb{T}_{a_{j}}} j^{-\alpha / 2 \beta}\left|S_{u}-\ell_{a_{j}}\right| \rightarrow 0 \text { as } j \rightarrow+\infty .
$$


Notice that

$$
\begin{aligned}
\sum_{j=1}^{\infty} \mathbb{E}_{\xi, \mathscr{F}_{a_{j}}}\left\{M_{j}\right\} & =\sum_{j=1}^{\infty}\left(\pi_{a_{j}}\right)^{-1} Z_{a_{j}} j^{-\alpha / 2 \beta} \mathbb{E}_{\xi, \mathscr{F}_{a_{j}}}\left\{\left|S_{u}-\ell_{a_{j}}\right|\right\} \\
& \leq \sum_{j=1}^{\infty} W_{a_{j}} j^{-\alpha / 2 \beta} \sqrt{\mathbb{E}_{\xi, \mathscr{F}_{a_{j}}}\left\{\left|S_{u}-\ell_{a_{j}}\right|^{2}\right\}} \\
& =\sum_{j=1}^{\infty} W_{a_{j}} j^{-\alpha / 2 \beta} a_{j}^{1 / 2} \sqrt{s_{a_{j}}^{2} / a_{j}}<\infty \text { a.s. }
\end{aligned}
$$

where $\mathbb{E}_{\xi, \mathscr{F}_{i}}(\cdot):=\mathbb{E}_{\xi}\left(\cdot \mid \mathscr{F}_{i}\right)$. Therefore $\sum_{j=1}^{\infty} M_{j}<\infty$ a.s. by the extended Borel-Cantelli Lemma. Then (3.15) follows.

Proof of Theorem 2.1. Theorem 2.1 is a direct consequence of Lemmas 3.1 and 3.2 .

\section{Proof of Theorem 2.2}

As in Section 2 we will need the decomposition $(3.2)$ but here we choose a new $\beta$ such that $1>\beta>\frac{1}{1+\eta}$ and then define $k_{n}$.

The proof of this theorem relies on Lemma 3.2 and the following lemma:

Lemma 4.1. If 2.1), 2.2 and 2.5 hold, then

$$
A_{n} \rightarrow 0 \quad \mathbb{P} \text {-a.s. }
$$

For the proof of Lemma 4.1, we shall use the following result on the the weighted moments for $W^{*}$ defined by $W^{*}=\sup _{n}\left\{W_{n}\right\}$.

Lemma 4.2. (14]) Assume that 2.1), 2.2 and 2.5 hold, then

$$
\mathbb{E}\left(W^{*}+1\right)\left(\ln \left(W^{*}+1\right)\right)^{1+\eta}<\infty .
$$

Furthermore, for $\beta>\frac{1}{1+\eta}$ and $\left\{r_{n}\right\}$ with $\liminf _{n \rightarrow \infty} \frac{\ln r_{n}}{n^{\beta}}>0$,

$$
\sum_{n=1}^{\infty} \mathbb{E}\left[\left(W^{*}+1\right) \mathbf{1}_{\left\{W^{*}+1 \geq r_{n}\right\}}\right]<+\infty .
$$

Proof of Lemma 4.1. We shall follow the notations defined in the proof of Lemma 3.1 .

By the extended Borel-Cantelli Lemma, we only need to prove that $\forall \varepsilon>0$,

$$
\sum_{n=1}^{\infty} \mathbb{P}_{k_{n}}\left(\left|A_{n}\right|>\varepsilon\right)<\infty
$$

As $\frac{Z_{k_{n}}(\mathbb{R})}{\pi_{k_{n}}} \rightarrow W>0$ a.s., then we shall prove that $\forall \varepsilon>0$,

$$
\sum_{n=1}^{\infty} \mathbb{P}_{k_{n}}\left(\left|Y_{n}\right|>\varepsilon\right)<\infty .
$$

We will get 4.5 if we can prove that the three terms in 3.6 are summable. We deal with them in three steps. We remind that $X_{n, u} \leq W_{n-k_{n}}(u)+1=W_{n-k_{n}} \circ\left(\theta^{|u|} \xi\right)+1$ for $u \in \mathbb{T}_{k_{n}}$.

Step 1. We first prove that

$$
\sum_{n=1}^{\infty} \mathbb{P}_{k_{n}}\left(Y_{n} \neq \bar{Y}_{n}\right)<\infty
$$


We observe that

$$
\mathbb{P}_{k_{n}}\left(Y_{n} \neq \bar{Y}_{n}\right) \leq\left[\mathbb{E}\left(\left(W_{n-k_{n}}+1\right) \mathbf{1}_{\left\{W_{n-k_{n}}+1 \geq r_{n}\right\}}\right)\right]_{r_{n}=Z_{k_{n}}} \leq\left[\mathbb{E}\left(\left(W^{*}+1\right) \mathbf{1}_{\left\{W^{*}+1 \geq r_{n}\right\}}\right)\right]_{r_{n}=Z_{k_{n}}} .
$$

Notice that $r_{n}=Z_{k_{n}}$ satisfies the condition $\liminf _{n \rightarrow \infty} \frac{\ln r_{n}}{n^{\beta}}>0$, because $Z_{k_{n}} / \pi_{k_{n}} \rightarrow W>0$ a.s., $\left(\pi_{n}\right)^{\frac{1}{n}} \rightarrow \exp \left\{\mathbb{E} \ln m_{0}\right\}$ a.s. and $k_{n} \sim n^{\beta}$. From this and Lemma 4.2 , we obtain that

$$
\sum_{n=1}^{\infty}\left[\mathbb{E}\left(\left(W^{*}+1\right) \mathbf{1}_{\left\{W^{*}+1 \geq r_{n}\right\}}\right)\right]_{r_{n}=Z_{k_{n}}}<+\infty
$$

Then 4.6 follows.

Step 2. We next prove that $\forall \varepsilon>0$,

$$
\sum_{n=1}^{\infty} \mathbb{P}_{k_{n}}\left(\left|\bar{Y}_{n}-\mathbb{E}_{\xi} \bar{Y}_{n}\right|>\varepsilon\right)<\infty
$$

Observe that $\forall u \in \mathbb{T}_{k_{n}}, n \geq 1$,

$$
\begin{aligned}
\mathbb{E}_{k_{n}} \bar{X}_{n, u}^{2} & =\mathbb{E}_{k_{n}}\left(X_{n, u}^{2} \mathbf{1}_{\left\{\left|X_{n, u}\right|<Z_{\left.k_{n}\right\}}\right.}\right) \\
& =\int_{0}^{\infty} 2 y \mathbb{P}_{k_{n}}\left(\left|X_{n, u}\right| \mathbf{1}_{\left\{\left|X_{n, u}\right|<Z_{k_{n}}\right\}}>y\right) d y \\
& \leq \int_{0}^{Z_{k_{n}}} 2 y \mathbb{P}_{k_{n}}\left(\left|X_{n, u}\right|>y\right) d y \\
& \leq \int_{0}^{Z_{k_{n}}} 2 y \mathbb{P}_{k_{n}}\left(\left|W_{n-k_{n}}(u)+1\right|>y\right) d y \\
& =\int_{0}^{Z_{k_{n}}} 2 y \mathbb{P}\left(\left|W_{n-k_{n}}+1\right|>y\right) d y \\
& \leq \int_{0}^{Z_{k_{n}}} 2 y \mathbb{P}\left(\left|W^{*}+1\right|>y\right) d y .
\end{aligned}
$$

Then we have

$$
\begin{aligned}
\sum_{n=1}^{\infty} \mathbb{P}_{k_{n}}\left(\left|\bar{Y}_{n}-\mathbb{E}_{\xi} \bar{Y}_{n}\right|>\varepsilon\right) & =\sum_{n=1}^{\infty} \mathbb{E}_{k_{n}} \mathbb{P}_{\xi, k_{n}}\left(\left|\bar{Y}_{n}-\mathbb{E}_{\xi, k_{n}} \bar{Y}_{n}\right|>\varepsilon\right) \\
& \leq \varepsilon^{-2} \sum_{n=1}^{\infty} \mathbb{E}_{k_{n}}\left(Z_{k_{n}}^{-2} \sum_{u \in \mathbb{T}_{k_{n}}} \mathbb{E}_{\xi, k_{n}} \bar{X}_{n, u}^{2}\right)=\varepsilon^{-2} \sum_{n=1}^{\infty}\left(Z_{k_{n}}^{-2} \sum_{u \in \mathbb{T}_{k_{n}}} \mathbb{E}_{k_{n}} \bar{X}_{n, u}^{2}\right) \\
& \leq \varepsilon^{-2} \sum_{n=1}^{\infty} \frac{1}{Z_{k_{n}}} \int_{0}^{Z_{k_{n}}} 2 y \mathbb{P}\left(\left|W^{*}+1\right|>y\right) d y \\
& =\varepsilon^{-2}\left[c_{2}+\int_{1}^{\infty}\left(\sum_{n=1}^{\infty} \frac{1}{Z_{k_{n}}} \mathbf{1}_{\left\{y<Z_{k_{n}}\right\}}\right) \cdot 2 y \mathbb{P}\left(\left|W^{*}+1\right|>y\right) d y\right] \\
& \leq \varepsilon^{-2}\left[c_{2}+\int_{1}^{\infty}\left(c_{3}+c_{4}(\ln y)^{1 / \beta-1}\right) \mathbb{P}\left(\left|W^{*}+1\right|>y\right) d y\right] \\
& \leq \varepsilon^{-2}\left(c_{2}+c_{5} \mathbb{E}\left(W^{*}+1\right) \ln ^{\eta}\left(W^{*}+1\right)\right)<\infty,
\end{aligned}
$$

where in the second line we have used Chebyshev's inequality, in the last two inequalities we use the results on $\left\{Z_{k_{n}}\right\}$ and the constant $c_{2}$ depends only on $\left\{Z_{k_{n}}\right\}$. Hence 4.8$]$ is proved.

Step 3. We then prove that $\forall \varepsilon>0$,

$$
\sum_{n=1}^{\infty} \mathbb{P}_{k_{n}}\left(\left|\mathbb{E}_{\xi} \bar{Y}_{n}\right|>\varepsilon\right)<\infty
$$


With the same procedure as in step 3 of the proof of Lemma 3.1 we can show that

$$
\begin{aligned}
\mathbb{P}_{k_{n}}\left(\left|\mathbb{E}_{\xi, k_{n}} \bar{Y}_{n}\right|>\varepsilon\right) & \leq \varepsilon^{-1} \mathbb{E}\left[\left|W_{n-k_{n}}+1\right| \mathbf{1}_{\left\{\left|W_{n-k_{n}}+1\right| \geq r_{n}\right\}}\right]_{r_{n}=Z_{k_{n}}} \\
& \leq \varepsilon^{-1} \mathbb{E}\left[\left|W^{*}+1\right| \mathbf{1}_{\left\{\left|W^{*}+1\right| \geq r_{n}\right\}}\right]_{r_{n}=Z_{k_{n}}}
\end{aligned}
$$

Combining this with 4.7), we prove 4.9).

Then the lemma has been proved.

Proof of Theorem 2.2. Theorem 2.2 is a direct consequence of Lemmas 4.1 and 3.2 .

\section{Proof of Theorem 2.3}

In this section, we give a proof of Theorem 2.3. using a result of Klebaner (1982, [13) on a branching random walk in a varying environment.

Proof of Theorem 2.3. We only need to prove that for almost every $\xi$,

$$
\left(\pi_{n}\right)^{-1} Z_{n}\left(\ell_{n}+s_{n} t\right) \rightarrow \Phi(t) W \text { a.s. under } \quad \mathbb{P}_{\xi}
$$

By Theorem 2 of Klebaner (1982, [13]), it suffices to verify the following two conditions for a.e. $\xi$ :

$$
\begin{aligned}
& \text { (I) } G_{0} * \cdots * G_{n-1}\left(\ell_{n}+s_{n} t\right) \rightarrow \Phi(t) \text { and } \frac{\sum_{k=0}^{n-1} \sigma_{k}^{2}}{n} \rightarrow \sigma^{2} \text { as } n \rightarrow \infty ; \\
& \text { (II) } \sum_{n=1}^{\infty} \frac{n^{\delta}}{\pi_{n}}<\infty \text { and } \sum_{n=1}^{\infty} \frac{\gamma_{n}^{2} n^{\delta+1}}{m_{n}^{2} \pi_{n+1}}<\infty \text { for some } \delta>2 .
\end{aligned}
$$

The first condition in (I) can be verified using the method in the proof of Lemma 3.3 and the second in (I) follows from the Birkhoff ergodic theorem.

We now check (II). Note that by calculus and the Birkhoff ergodic theorem,

$$
\lim _{n \rightarrow+\infty}\left(\frac{n^{\delta}}{\pi_{n}}\right)^{1 / n}=\frac{\lim _{n \rightarrow+\infty}(\sqrt[n]{n})^{\delta}}{\lim _{n \rightarrow+\infty}\left(\pi_{n}\right)^{1 / n}}=\exp \left\{-\mathbb{E} \ln m_{0}\right\}<1 \text { a.s. }
$$

where the last inequality follows from 2.1. Thus the Cauchy's root test ensures the convergence of the first series in (II).

The left thing is to prove the convergence of the second series in (II).

By (2.7), we see that $\frac{\ln ^{+}\left(\gamma_{n}^{2} / m_{n}^{2}\right)}{n} \rightarrow 0$ a.s.. Then by the Birkhoff ergodic theorem,

$$
\begin{aligned}
& \lim _{n \rightarrow \infty}\left(\frac{\gamma_{n}^{2}}{m_{n}^{2} \pi_{n+1}}\right)^{1 / n}=\lim _{n \rightarrow \infty} \exp \left\{\frac{\ln \left(\gamma_{n}^{2} / m_{n}^{2}\right)-\ln \pi_{n+1}}{n}\right\} \\
\leq & \exp \left\{\lim _{n \rightarrow \infty} \frac{\ln ^{+}\left(\gamma_{n}^{2} / m_{n}^{2}\right)}{n}-\mathbb{E} \ln m_{0}\right\}=\exp \left\{-\mathbb{E} \ln m_{0}\right\}<1 \text { a.s.. }
\end{aligned}
$$

Now the Cauchy's root test shows the convergence of the second series in (II).

Therefore by Theorem 2 in [13, 5.1) holds; using the dominated convergence theorem again, we see that 2.8 holds.

\section{Acknowledgements}

The project is partially supported by National Nature Science Foundation of China (Grant No. 11101039) and the Research Fund for the Doctoral Program of Higher Education (Grant No. 20100003110004). 


\section{References}

[1] S. Asmussen and N. Kaplan, Branching random walks. I, Stochastic Processes Appl. 4 (1976), no. $1,1-13$.

[2] K. B. Athreya and S. Karlin, On branching processes with random environments. I. Extinction probabilities, Ann. Math. Statist. 42 (1971), 1499-1520.

[3] K. B. Athreya and S. Karlin, On branching processes with random environments. II. Limit theorems, Ann. Math. Statist. 42 (1971), 1843-1858.

[4] J.-B. Baillon, Ph. Clément, A. Greven, and F. den Hollander, A variational approach to branching random walk in random environment, Ann. Probab. 21 (1993), no. 1, 290-317.

[5] J. D. Biggins, Martingale convergence in the branching random walk, J. Appl. Probability 14 (1977), no. 1, 25-37.

[6] J. D. Biggins, The central limit theorem for the supercritical branching random walk, and related results, Stochastic Process. Appl. 34 (1990), no. 2, 255-274.

[7] J. D. Biggins and A. E. Kyprianou, Measure change in multitype branching, Adv. in Appl. Probab. 36 (2004), no. 2, 544-581.

[8] R. Durrett, Probability: theory and examples, second ed., Duxbury Press, Belmont, CA, 1996.

[9] A. Greven and F. den Hollander, Branching random walk in random environment: phase transitions for local and global growth rates, Probab. Theory Related Fields 91 (1992), no. 2, $195-249$.

[10] T. E. Harris, The theory of branching processes, Die Grundlehren der Mathematischen Wissenschaften, Bd. 119, Springer-Verlag, Berlin, 1963.

[11] N. Kaplan, A note on the branching random walk, J. Appl. Probab. 19 (1982), no. 2, 421-424.

[12] N. Kaplan and S. Asmussen, Branching random walks. II, Stochastic Processes Appl. 4 (1976), no. $1,15-31$.

[13] C. F. Klebaner, Branching random walk in varying environments, Adv. in Appl. Probab. 14 (1982), no. 2, 359-367.

[14] X. Liang, Propriétés asymptotiques des martingales de Mandelbrot et des marches aléatoires branchantes, doctor thesis, Université de Bretagne-Sud, France (2010).

[15] Q. Liu, Branching random walks in random environment, Proceedings of the 4th International Congress of Chinese Mathematicians (ICCM 2007) (L. Ji, K. Liu, L. Yang, and S.-T. Yau, eds.), vol. II, 2007, pp. 702-219.

[16] V. V. Petrov, Limit theorems of probability theory, Oxford Studies in Probability, vol. 4, The Clarendon Press Oxford University Press, New York, 1995, Sequences of independent random variables, Oxford Science Publications.

[17] P. Révész, Random walks of infinitely many particles, World Scientific Publishing Co. Inc., River Edge, NJ, 1994.

[18] W. L. Smith and W. E. Wilkinson, On branching processes in random environments, Ann. Math. Statist. 40 (1969), 814-827. 